\title{
Living with childhood asthma: parental perceptions of risk in the household environment and strategies for coping
}

\author{
Ann Crosland ${ }^{1,2}$, Isabel Gordon ${ }^{1,2}$ and Annette Payne ${ }^{3}$ \\ ${ }^{1}$ Primary Care Development Centre, Northumbria University, UK \\ ${ }^{2}$ Centre for Primary and Community Care, School of Health, Social and Natural Sciences, University of Sunderland, \\ Sunderland, UK \\ ${ }^{3}$ Newcastle Primary Care Trust, Newcastle upon Tyne, UK
}

\begin{abstract}
Aim: To explore parents' perceptions of environmental household risks to their child's asthma and to identify the strategies they adopt in relation to these perceived risks.

Background: The prevalence of childhood asthma is increasing worldwide and especially in the UK. Asthma is more common in areas of socio-economic disadvantage. Household environmental factors have been implicated in some of this increase. A number of factors in the home environment have been found to act as triggers to asthma symptoms, including high humidity levels, poor ventilation, mould, second-hand tobacco smoke and pet allergens. Little is known about how parents, as the main care-givers and decision makers in the home, perceive and cope with the risks posed by these triggers. Methods: Semi-structured interviews were conducted with a purposive sample of parents of 32 children with asthma aged 4 to 16 years and living in a socio-economically disadvantaged urban community in the North East of England. Interviews were audiotaped, transcribed verbatim and analysed using constant comparison techniques. Findings: All parents were aware of some of the risks their children faced at home. Some appeared to know more than others and coping styles varied. A typology of three groups of parents was identified: those who actively seek advice and adopt clear preventative strategies (preventers); those who minimize the risks and only react when things go wrong (reactors); and those who engage in compensatory activities in an attempt to trade-off between harms and benefits (compensators). The unifying themes underpinning these different styles are that all parents are motivated to maintain normal family life but that they adopt different strategies to achieve this.
\end{abstract}

Key words: childhood asthma; parental attitudes; household risks; primary care

Received: 31 July 2008; accepted: 5 December 2008

\section{Introduction}

The prevalence of childhood asthma is increasing throughout the developed world, particularly in

Correspondence to: Professor Ann Crosland, Centre for Primary and Community Care, School of Health, Social and Natural Sciences, Sciences Complex, University of Sunderland, Wharncliffe Street, Sunderland SR1 3SD, UK. Email: ann.crosland@sunderland.ac.uk

(C) 2009 Cambridge University Press the UK, which has one of the highest rates of childhood asthma in the world. It is estimated that 1 in 10 children in the UK now have the disease (Anderson et al., 2004; Richardson et al., 2005), and that rates in seven- and eight-year olds increased by $5 \%$ between 1991 and 2002 (Butland et al., 2006). Asthma is more common in boys (Diette et al., 2007) and in those living in socioeconomically disadvantaged communities ( $\mathrm{Wu}$ and Takaro, 2007). The aetiology of the disease is 
complex but is known to involve an interplay between genetic and environmental factors (Jones, 1998). As most people today spend between $90 \%$ and $95 \%$ of their time indoors, the indoor environment can play a significant part in triggering or exacerbating asthma symptoms. It is estimated that up to $66 \%$ of time is spent at home and that this percentage is likely to be higher among pre-school children (Leech et al., 2002). Modern household living conditions that incorporate higher levels of insulation, central heating and poor air circulation are implicated in the increased rates of childhood asthma in the UK (Lindfors et al., 1995; Jones, 1998; Richardson et al., 2005). Previous studies have shown that a number of factors in the home environment can act as triggers to asthma. High humidity levels, poor ventilation, mould, house dust mites, environmental tobacco smoke and pet allergens have all been identified as factors that can exacerbate pre-existing symptoms in asthmatic children (Lindfors et al., 1995; Richardson et al., 2005; Robinson and Kirkaldy, 2007; Vork et al., 2007).

Most children have little control of their home environment and are dependent on parental decisions to help minimize the risks they face. PetersonSweeney et al. (2002) suggest that the only effective way that the risks associated with childhood asthma can be minimized is through effective partnerships and communication between parents and health care providers (Peterson-Sweeney et al., 2003). All need a common understanding of the nature of the disease and of the factors in a household environment that can trigger an asthma episode if it is to be controlled and attacks prevented.

A review of household intervention studies aimed at improving health outcomes for asthmatic children showed that whilst mechanical interventions to reduce environmental triggers (such as house dust mites) had limited health benefits, educational interventions with parents showed no benefits whatsoever. Combining the two approaches yielded slightly better results, but indicated that research is needed to help understand the context within which these interventions are carried out if the problems posed by triggers to asthma in the household environment are to be tackled (Wu and Takaro, 2007). Hunter et al. (2003) go further and suggest that future research should be considered within the context of perceptions and socio-behavioural factors if reductions in childhood asthma are to be achieved (Hunter et al., 2003).

A number of studies have sought to elicit levels of parental understanding and beliefs about the disease (Cane et al., 2001), how parental views compare with those of their children (Callery et al., 2003) or health professionals (Horky et al., 2007), their understanding and attitudes to treatments (Peterson-Sweeney et al., 2003) and how they cope with the burden of the disease (Mallick et al., 1994). These studies highlight that parents are less likely than health professionals to recognize asthma as a chronic disease (Horky et al., 2007), that they struggle to manage their child's emotional support needs (Svavasdottir et al., 2000) and that they can be resistant to social discourses that do not fit with their own existing theories, for example the link between smoking and asthma is commonly rejected by smoking mothers who seek alternative explanations such as genetic arguments to support continuation of their behaviour.

Research with asthmatic adults has sought explanatory models for the way they cope with or manage the disease. Adams et al. (1997) identified two groups, plus a third subgroup, in relation to people's attitudes to medication and how they adapt it to their everyday lives. Here, differences were based on contrasting beliefs about the nature of the disease and readiness to accept an identity of asthma (Adams et al., 1997). These groups of acceptors, deniers and pragmatists (a subgroup of the acceptors) are very similar to two of the groups outlined by Carver et al. (1989) in their Coping Orientations to Problems Experienced (COPE) model of adaptation to chronic illness. These models suggest that without an understanding of where people are in terms of their beliefs about the disease and their preparedness for change, it is impossible to develop appropriate support and advice (Svavasdottir et al., 2000). Given the limited information available about parental perceptions of asthma in general and especially information about parental views of risks children face in the home, this study sought to explore parents' perceptions of environmental household risks to their child's asthma and the strategies they adopt in relation to these perceived risks. The longer-term aim was to provide contextual information that can inform future primary care services and education for parents of children with asthma. 


\section{Methods}

In-depth qualitative interviews were carried out by IG as part of a larger study evaluating the impact on families of a pilot intervention to eradicate dust mite load in the homes of children aged 4 to 16 years with asthma, living in a socioeconomically disadvantaged community. Interviews were carried out with a purposive sample of the parents of 32 children with asthma who were involved in the evaluation of the pilot programme. A total of 30 interviews were carried out with parents three months after they had the intervention installed (one parent had two children enrolled in the study). All of the parents lived in the same inner city area of Newcastle. In some families, one parent was present at interview (22 mothers and two fathers), while in the remaining six interviews both parents were present and contributed. In 12 of the interviews (six single-parent and all six couple interviews), the child with asthma was also present. Interviews explored parents' perceptions of risks at home, their experiences of coping, and strategies they adopted to manage their child's asthma. Ethical approval was obtained from Newcastle and North Tyneside Research Ethics Committee.

All interviews were audiotaped and transcribed verbatim. Analysis followed the method of constant comparison where responses from early interviews were incorporated into the topics discussed in later interviews (Strauss and Corbin, 1998). At regular stages throughout the interview study, all authors read transcripts and met to discuss and develop themes. These themes were then developed into the model presented here.

\section{Findings}

All parents recognized that factors at home and the wider environment could act as a trigger to asthma attacks in their children. The most commonly cited triggers were smoking, pet hair and house dust. Parents perceived some triggers to their child's asthma as unavoidable and not in their control; these were triggers in the external environment such as the weather or pollen. Other triggers such as smoking, keeping pets, exercise, opening windows, use of central heating and use of household cleaning or decorating products were seen to be much more avoidable and most parents felt they had a greater element of control over these. All recognized at least some of these; however, few parents could list more than three, indicating variation in awareness, knowledge and understanding of the disease. Whilst all parents recognized that they had a role to play in minimizing the effects of environmental triggers, their ways of coping and strategies they adopted varied. Closer inspection of this variation revealed three distinct groups of preventers, reactors and compensators. The group of preventers was the smallest group and accounted for seven of the interviews. The other two groups were similar in size with 11 and 12 interviews falling into these categories.

\section{Preventers}

The distinguishing feature of preventer parents was that they actively sought out information about asthma from a variety of sources. Sources of information included health professionals and the media, the latter of which included newspapers, TV programmes or the internet. This group was most likely to seek professional advice and had the most contact with primary care professionals. They were constantly seeking new information and were prepared to consider any advice that could help improve their child's symptoms:

Once we found out that (Name)'s allergy was to the dust mite I then wanted to know more about the dust mite, so I got the leaflet and I sort of went on line and had a look.

(Mother, Family 5; Mother and son interviewed)

If it's going to make it better for him I would try anything.

$$
\text { (Mother, Family 4; son present) }
$$

Active advice-seeking provided a source of coping, which was then translated into the use of preventative strategies. Such strategies included acting on advice to clean the house regularly or wash laundry at recommended temperatures. This group of parents was also most likely to encourage the use of preventative medication. They were also most likely to encourage their asthmatic child to be physically active to help 
build their strength and to try and prevent serious attacks:

I push them to do all the sports is it will build their lungs up rather than them just sitting as couch potatoes and then the next thing you know they've got to run down the street and cannot even run.

(Mother, Family 16)

They were often worried about the severity of their child's asthma and what might happen if they did not make all efforts to prevent asthma attacks. In so doing, wherever possible they avoided known triggers such as second-hand smoke or pet hairs.

In a minority of cases this level of worry and preventive behaviour was taken to extremes, in particular in relation to household cleaning. The small number of parents, particularly mothers, who reported being anxious in this way attributed previous severe attacks to not having cleaned thoroughly enough. It appeared with this group that taking preventive action helped them assume a sense of control over the situation. However, if their preventive action then failed it could leave them with a sense of personal failure:

I felt I probably hadn't cleaned enough so I sort of set to...pulling the books out and wiping the tops so I felt I had... maybe I was just being sensitive about not having cleaned thoroughly enough.

(Mother, Family 5; son present)

\section{Reactors}

As their name suggests, reactors were most likely to wait until an asthma attack occurred before taking action to minimize its effects. They were less likely to seek professional advice than the preventers and when advice was given they did not always take it on board:

When we went to the appointment we worked out he had been given the inhalers all the wrong way. All that time. And this was the first time it was picked up on, and I still think even now that we are giving the inhalers the wrong way. I still think we are doing it wrong.

(Mother, Family 23)

This group reported mixed messages from professionals and indeed often demonstrated a lack of trust in the advice available. They commonly reported that they believed doctors know little about the disease. They generally did not appear to pay much attention to their child's asthma until a problem arose preferring to rely on their own skills and common sense knowledge of asthma when necessary:

I notice like if you talk to one doctor the idea is totally different to another doctor then you've got another doctor that will tell you a totally different idea, ad you're like, wow okay.

(Mother, Family 24; Daughter present)

Sometimes I feel that they [the doctors] don't know enough about it.

(Mother, Family 31)

I probably know more about childhood asthma than half the doctors do.

(Mother, Family 26; son present)

In some cases there was a sense of preferring not to know too much about triggers to their asthma as the following parent illustrates when talking about house dust mites:

No don't want to know, bad enough looking at the picture without finding out what they actually do as well.

(Mother, Family 3; couple interview)

Reactor parents developed coping strategies through a process of trial and error. Examples of strategies adopted included hot baths, using chest rubs and keeping their child off school should an attack occur:

I just do, like, what I've always done, if they are bad I keep them in do you know what I mean, lie them on the settee just give them Calpol or Paracetamol and use the em, the smelly for them.

(Mother, Family 27; son present)

These parents were more likely than others to rely on the use of reliever medication and were often confused about what the 'brown' preventer inhaler was for:

He's only been taking the blue one like when and if needed. He's never really needed his brown one.

(Mother, Family 8) 
It's something I've never really thought about till now but we're not taking the brown one [inhaler] constantly.

(Father, Family 2; couple interview)

As a group, reactors were much more instinctive in their reactions and had a tendency to minimize the risks to their children. In so doing, they tended to deal with problems as they arose rather than actively avoid them. They were more likely to see their children's asthma as a mild form of the disease, despite little evidence to indicate that their children's asthma was less serious than that of children of parents with other coping styles:

I've never needed any support, its just very mild asthma and it doesn't really get in the way of his daily living so, not that bothered.

(Mother, Family 32)

It [the asthma] is not regular, its not, it just depends... I don't know what sets it off.

(Mother, Family 25; Mother, couple interview)

\section{Compensators}

The largest group of parents fell into the category of compensators. As with the other two groups, this group recognized the role of environmental triggers in general and triggers within the home environment in particular. However, rather than avoid recognized triggers such as smoking or keeping pets, they talked about compensatory activities. So pets may be kept downstairs away from the child's bedroom and pet hairs removed at regular intervals, smoking may be restricted to one part of the house, or cleaning used to compensate for smoking:

I don't smoke everywhere in me house. If I was like sitting and smoking on top of him I would say it's my fault, you know, but there is none of that...this is just a free household and I keep on top of me polishing and me hovering, and I always have done.

(Mother, Family 20; son present)

Whilst some recognized that they were exposing their children to potentially harmful triggers to their asthma, there was a sense of denial amongst some others as to the harmful potential of this exposure. For example, one parent reported that their child's asthma was triggered by dog hair and even though they had a dog in the house they reported it was only other people's dogs who posed a risk; another reported only smoking menthol cigarettes, or only smoking indoors while their child was out of the house:

He's not [allergic] to the dog we've got, but he is allergic to my sister's dog.

(Mother, Family 21)

I've stopped smoking, I buy the 10 menthol and I think that's helped a lot as well.

(Mother, Family 22)

Like the reactors, this group did not actively seek information or advice, but when faced with information, attempted a trade-off between what they perceived to be harmful activities and those they perceived to be helpful:

I knew a lot of it [the asthma] was to do with cats, and we have got a cat mind but that's not allowed upstairs anymore.

(Father, Family 15)

And I've stopped smoking.... a had one tab this morning but that was when he was at school.

(Mother, Family 22)

This group appeared to prioritize information that supported their existing behaviour patterns and that helped them to balance the needs of their asthmatic child with those of other family members. Where possible, they appeared to avoid messages that contradicted these priorities.

\section{Maintaining normal family life}

Each of the three groups of parents differs in the judgements made in the management of their child's asthma, their views about advice and information seeking, their preventative behaviour, and their attitudes to potentially harmful triggers. However, all groups were unified in their motivation to maintain normal family life. Each group adopted a different approach to achieve this: preventers were very proactive in their approach to manage asthma and to minimize the risks posed to their child and in so doing they sometimes adapted normal family life to the needs of the asthmatic child; reactors carried on as normal by minimizing, not only the impact of triggers in the home environment on their child's 
health but also the severity of the symptoms their child experienced; compensators combined some elements of both of the other two groups in so far as they made efforts to minimize the impact of those triggers they considered to be important through compensatory activities and at the same time denying the effects of others.

\section{Discussion}

The main strength of this study is that it provides an in-depth contextualized account of parent's views and self-reported strategies for managing their child's asthma. It is carried out with a social group amongst whom childhood asthma levels are high (Wu and Takaro, 2007) and highlights that parents are generally aware of triggers to asthma in the household environment but interpret this information differently and develop different coping strategies. The main limitations are that the findings rely on self-reported strategies for coping with asthma in a group of white working class parents living in a socio-economically disadvantaged community in the North East of England and may not therefore be transferable to other groups from different backgrounds or geographical areas. The study was carried out with parents and although some children were present during the interview their views were not sought directly. Guyatt et al. (1997) reported that children's accounts of asthma differ from those of care-givers, especially when considering the impact of the disease on quality of life, and also that care-givers are better at assessing improvements in symptoms. The authors of the present study recognize that children and young people may be able to offer a different account and also that most decision-making in the home environment will fall to parents and care-givers rather than to children themselves.

Those who actively seek advice appear most likely to follow it whilst those who are less proactive, pick and choose what information to follow, suggesting that strategies that seek to educate parents through the provision of better information are only likely to succeed with a minority of committed parents. Wu and Takaro (2007) found that trials of educational interventions with parents have no proven effectiveness in reducing asthma in children. The findings here suggest a more tailored approach might be necessary.
Whilst much is known about factors at home that can prevent exacerbation of existing asthma, not all parents appear to follow the advice available. This cannot be explained purely by lack of awareness or knowledge, but rather by how parents seek out and rationalize the information and choose the options available to them. Other influences include beliefs about the knowledge and effectiveness of health professionals and lack of understanding of the role of preventative medication. In this study, there was considerable variation in beliefs about professional knowledge of asthma with reactors in particular demonstrating little faith in the knowledge and awareness of doctors. This contrasts with a previous study that compared the views of parents and health care providers and concluded that parents had more faith in the abilities of health care providers to manage childhood asthma than did the providers themselves (Horky et al., 2007).

Other studies have explored parental attitudes to medication and concluded that parents are concerned about the use of medication every day and that people may be predisposed not to take medication (Cane et al., 2001). Others have found that those with diminished treatment expectations are the most likely not to adhere to medication treatment regimens (Yoos et al., 2003) and that those who assess asthma severity through the absence of acute attacks are more likely to manage the disease as intermittent acute episodes, and not as a long-term condition requiring preventative strategies (Callery et al., 2003). Such findings help explain the reluctance of reactors and compensators to encourage their children to take prophylactic medication and furthermore suggest that consistent use of preventive medication is the exception rather than the norm.

Attitudes to other potentially harmful triggers such as smoking have been explored elsewhere; for example, Robinson and Kirkaldy (2007) also found that whilst mothers are aware of the messages about the links between smoking and childhood illness, they tend to seek out alternative explanations over which they have little control as a way of rationalizing their own continued smoking. The findings here, especially relating to the compensator group, support this theory and add to it by identifying other triggers such as pet hair being rationalized in the same way. This supports the proposition here that parents 
are primarily motivated to maintain the status quo and continue with family life.

Comparing the findings presented in this study with those of Adams et al. (1997), who looked at the attitudes of adults with asthma, there are a number of similarities, in particular between Adams' group of deniers and the reactors outlined above in so far as both fail to recognize the severity of symptoms and are motivated not to take preventative action. However, the reactors tended to minimize the impact of asthma rather than deny it altogether. Furthermore, the other two groups differed in their perspective from the groups Adams et al. presented. Preventers may appear to accept the diagnosis of asthma, which accords with Adams' acceptors; however, they do not necessarily consider it to be part of their family identity. There is no evidence that compensators bear any consistent relationship with any of Adams' groups. Perhaps the main difference between the two studies is in the overall themes that explained the motivations of parents and those of adults with asthma. Adams concluded that the concept of identity and the challenges being asthmatic posed to this was central to the treatment choices and behaviours of adults with asthma (Adams et al., 1997). In this study, having an asthmatic child does not appear to be important to parental or family identity but rather maintaining family life and normality appears to be central to the choices parents make.

\section{Implications}

The findings presented here suggest that models of support for parents with asthmatic children that focus on information-giving in isolation from other forms of support are overly simplistic. It seems that there is a need to understand how parents make choices through a trade-off between: awareness; knowledge of possible alternatives; their personal preferences and those of powerful others, assessment of risks; and maintaining a balance between the needs of the asthmatic child and those of the family as a whole. The findings suggest that in providing support and advice to parents, one approach will not fit all, and that there is a need to recognize different coping styles, that people react differently and to target advice and support according to needs.
The implications for each of the groups outlined above are as follows:

- Preventers may be recognized by their proactive advice-seeking and desire for support, and may also appear the most anxious group of parents. Support for this group would include making information and advice easily accessible via a range of different media, recognizing and supporting their preventative actions but making sure that they do not blame themselves if their child becomes ill, and helping them to recognize signs of stress and provide additional support for this.

- Reactors are recognizable by their minimization of the severity of the symptoms their child experiences. They are most likely to seek help only during an acute episode of the illness. They may benefit from support or advice given by people they perceive to be experts, that is other parents of asthmatic children in communitybased rather than health care settings. Checking out how much they know about medication and whether they know how and when medication should be taken would be an important means of support, as would provision of advice on how and when to seek help in an emergency.

- Compensators are most likely to recognize health-related messages but to re-orient these to their own needs. They may be difficult to identify other than by asking them about what strategies they adopt to minimize the environmental triggers their children face at home. Strategies for support would include discussing compensatory behaviour and how it impacts upon their child; for example, rather than telling people not to smoke, advise them that if they must do so how they could do this to minimize the risk to their children.

\section{Conclusion}

This study has been carried out with a small, urban, mainly white-working class sample of parents living in the North East of England and may need to be tested with other groups to assess the transferability of the findings. The main findings from the study are that parents, motivated by a desire to maintain normal family life, make different judgements in the management of 
childhood asthma at home. The main differences relate to advice seeking and utilization of information, preventative strategies and attitudes to potentially harmful triggers. Different strategies are therefore needed to provide advice and support for each of these different groups of parents.

\section{Acknowledgements}

This study was funded by Newcastle Primary Care Trust and National Energy Action. Due thanks to all participants and to the Newcastle Asthma Project Steering Group.

Ethical Approval for this study was obtained from Newcastle and North Tyneside Local Research Ethics Committee 2.

\section{References}

Adams, S., Pill, R. et al. 1997: Medication, chronic illness and identity: the perspective of people with asthma. Social Science and Medicine 45, 189-201.

Anderson, A., Ruggles, R. et al. 2004: Trends in prevalence of symptoms of asthma, hay fever, and eczema in 12-14 year olds in the British Isles 1995-2002. British Medical Journal 328, 1052-53.

Butland, B.K., Strachan, D.P. et al. 2006: Childhood asthma in South London: trends in prevalence and use of medical services 1991-2002. Thorax 61, 383-87.

Callery, P., Milnes, L. et al. 2003: Qualitative study of young people's and parents beliefs about childhood asthma. British Journal of General Practice 53, 185-90.

Cane, R., Pao, C. et al. 2001: Understanding childhood asthma in focus groups: Perspectives from mothers of different ethnic backgrounds. BMC Family Practice 2, 1-6.

Carver, C.S., Scheier, M.F. et al. 1989: Assessing coping strategies: a theoretically based approach. Journal of Personality and Social Psychology 56, 267-83.

Diette, G.B., Hansel, N.M. et al. 2007: Home indoor pollutant exposures among inner city children with and without asthma. Environmental Health Perspectives 115, 1665-69.

Guyatt, G.H., Juniper, E.F., Feeny, D.H., Griffith, L.E. and Ferrie, P.J. 1997: Children and adult perceptions of childhood asthma. Paediatrics 99, 165-68.
Horky, S.C., Kleinman, A.H. et al. 2007: A comparison of parent and provider beliefs about asthma in children. Pediatric Asthma, Allergy and Immunology 20, 36-46.

Hunter, P.R., Davies, M.A. et al. 2003: The prevalence of self reported symptoms of respiratory disease and community belief about the severity of pollution from various sources. International Journal of Environmental Health Research 13, 227-38.

Jones, A.P. 1998: Asthma and domestic air quality. Social Science and Medicine 47, 755-64.

Leech, J.A., Nelson, W.C., Burnett, R.T., Aaron, S. and Reizenne, M.E. 2002: It's about time: a comparison of Canadian and American time-activity patterns. Journal of Exposure Analysis and Environmental Epidemiology 12, 427-32.

Lindfors, A., Wickman, M. et al. 1995: Indoor environmental risk factors in young asthmatics: a case-control study. Archives of Disease in Childhood 73, 408-12.

Mallick, M., Holden, G. et al. 1994: Coping with childhood asthma. Health and Social Work 19, 103-11.

Peterson-Sweeney, K., McMullen, A. et al. 2003: Parental perceptions of the child's asthma: management and medication use. Journal of Pediatric Health Care 17, $118-25$.

Richardson, G., Eick, S. et al. 2005: How is the indoor environment related to asthma? A literature review. Journal of Advanced Nursing 52, 328-39.

Robinson, J. and Kirkaldy, A.J. 2007: "You think that I'm smoking and they're not": Why mothers still smoke in the home. Social Science and Medicine 65, 641-52.

Strauss, A. and Corbin, J. 1998: Basics of qualitative research: techniques and procedures for developing grounded theory. Thousand Oaks: Sage.

Svavasdottir, E., McCubbin, M. et al. 2000: Well-being of parents of young children with asthma. Research in Nursing and Health 23, 346-58.

Vork, K.L., Broadwin, R.L. and Blaisdell, R.J. 2007: Developing asthma in childhood from exposure to second hand tobacco smoke: insights from a meta-regression. Environmental Health Perspectives 115, 1394-1400.

Wu, F. and Takaro, T. 2007: Childhood asthma and environmental interventions. Environmental Health Perspectives 115, 971-75.

Yoos, H.L., Kitzman, H., McMullan, A. and Sidorak, K. 2003: Symptom perception in childhood asthma: how accurate are children and their parents? Journal of Asthma 40, 27-29. 